\title{
Aerodynamic repellency of impacting liquids
}

\author{
Anaïs Gauthier, Ambre Bouillant, Christophe Clanet, and David Quéré \\ Physique \& Mécanique des Milieux Hétérogènes, UMR 7636 du CNRS, ESPCI, 75005 Paris, France \\ and LadHyX, UMR 7646 du CNRS, École polytechnique, 91128 Palaiseau, France
}

(Received 22 June 2017; published 15 May 2018)

\begin{abstract}
Impacting liquids can be reflected by moving solid plates, provided the surface is fast enough. We describe and model here the threshold speed of bouncing, in particular as a function of the impact velocity of the incoming liquid. We also demonstrate that the aerodynamic force responsible for the nonwetting behavior induces an oblique rebound, which contributes to the liquid removal. In summary, this situation repels viscous, low surface tension drops of any size, all kinds of cases where repellency is impossible to achieve by other means.
\end{abstract}

DOI: 10.1103/PhysRevFluids.3.054002

\section{INTRODUCTION}

On many occasions, it is desirable to prevent rain, splashes or sprays from contacting solids [1], an ability of the so-called repellent materials that reflect impacting drops. The addition of hydrophobic textures, either natural [2-4] or artificial [5-7] makes the surfaces water repellent, and a careful choice of the textures permits us to extend this property to cohesive oils (superoleophobicity), provided that the impact velocity remains modest $[8,9]$. Nonwetting can also be induced by heating solids above the Leidenfrost temperature at which volatile liquids levitate above a vapor cushion $[10,11]$. Hence each of these solutions works for only a limited family of liquids, which must have in any case a low viscosity, comparable to that of water.

Dynamical tricks can also generate repellency. Oil bounces on a vibrating bath of the same oil provided that the amplitude and frequency of the vibration are carefully selected [12]. The effect is not observed on vibrating solids because the substrate must deform to prevent contact and further coalescence [13]. Yet quasistatic drops were shown to be able to levitate on flat conveyor belts moving at typically $1 \mathrm{~m} / \mathrm{s}[14,15]$ or above flowing liquids [16,17]. Levitation is nevertheless much more challenging for impacting drops, whose inertia is generally large enough to suppress the existence of a lubricating film of air. However, Povarov et al. reported in a pioneering study [1] that impacting water drops bounce on moving plates if the solid velocity exceeds a threshold value as high as typically $50 \mathrm{~m} / \mathrm{s}$ - speeds such that inertia dominates the viscous effects operating for quasistatic levitating drops $[14,15]$. Povarov et al. also showed that the minimum plate velocity $V^{*}$ at which drops are repelled sharply increases with the impact speed $U$ and proposed a model relating the speed $V^{*}$ to the interaction of the drop with the air boundary layer entrained by the plate. Here we question and develop this model and extend experiments to viscous and wetting liquids, also found to be repelled by moving plates. Observation of the transient drop deformation during impact allows us to derive an explicit formula for the threshold of repellency and to describe how the liquid gets carried away after successive rebounds. We finally discuss the case of submillimetric droplets, which are shown to be swept by moving plates even more efficiently than bigger drops, a behavior in stark contrast with classical observations on superhydrophobic surfaces [5].

\section{EXPERIMENT}

The setup is shown in Fig. 1(a). A drop with radius $R$ hits a rotating disk of polished aluminium with an impact velocity $U$. The disk is entrained by a dc motor with an angular velocity $\omega$ between 

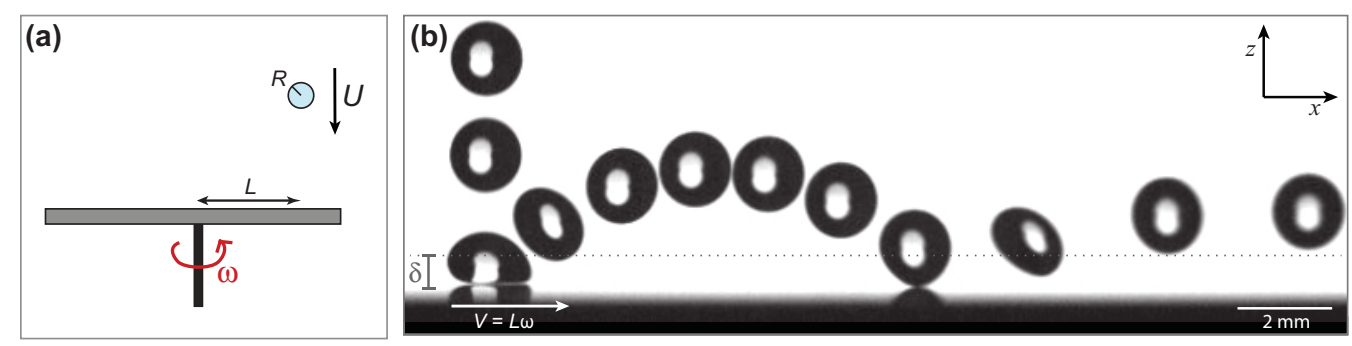

FIG. 1. (a) Experimental setup. A drop with radius $R$ impacts at velocity $U$ a rotating aluminum plate moving at $V=L \omega$ at the impact point. (b) Chronophotography showing successive images (separated by $6.6 \mathrm{~ms}$ ) of a drop of silicone oil $(R=0.75 \mathrm{~mm})$ impinging at $U=0.37 \mathrm{~m} / \mathrm{s}$ a plate moving at $V=17 \mathrm{~m} / \mathrm{s}$. The thickness $\delta$ of the boundary layer of air is indicated to scale with a dotted line. The corresponding movie is movie 1 , see Ref. [22].

0 and $500 \mathrm{rad} / \mathrm{s}$ so that the local surface speed $V=L \omega$ at the impact point (chosen at a distance $L=9 \mathrm{~cm}$ from the disk center) spans from 0 to $45 \mathrm{~m} / \mathrm{s}$, with a precision of $\pm 0.1 \mathrm{~m} / \mathrm{s}$. Drops are deposited close to the edge of the disk (of radius $10 \mathrm{~cm}$ ), so that the plate velocity below the drop does not vary by more than $10 \%$ while the liquid is carried away by centrifugal forces. The rotating disk entrains a laminar boundary layer of air with thickness $\delta \approx 2.5\left(\eta_{\mathrm{a}} / \rho_{\mathrm{a}} \omega\right)^{1 / 2}$, denoting $\eta_{\mathrm{a}}$ and $\rho_{\mathrm{a}}$ as the dynamic viscosity and density of air (at $25^{\circ} \mathrm{C}$, the temperature of experiments, $\eta_{\mathrm{a}}=20 \mu \mathrm{Pa} . \mathrm{s}$ and $\rho_{\mathrm{a}}=1.2 \mathrm{~kg} / \mathrm{m}^{3}$ ) [18-21]. Hence the boundary layer thickness decreases from $\delta \approx 1.6 \mathrm{~mm}$ at $V=5 \mathrm{~m} / \mathrm{s}$ to $\delta \approx 800 \mu \mathrm{m}$ at $V=20 \mathrm{~m} / \mathrm{s}$. The main liquid used in this study is a silicone oil (siloxy-terminated dimethyl siloxane, from Dow Corning) with viscosity $\eta=96 \mathrm{mPa} \mathrm{s}$, surface tension $\gamma=21 \mathrm{mN} / \mathrm{m}$ and density $\rho=960 \mathrm{~kg} / \mathrm{m}^{3}$. Drops with radius $R=0.5-1.5 \mathrm{~mm}$ are dispensed from calibrated needles located at various distances from the plate, allowing us to tune the impact velocity $U$ from $10 \mathrm{~cm} / \mathrm{s}$ to $1 \mathrm{~m} / \mathrm{s}$. Smaller drops with $R=0.1-0.5 \mathrm{~mm}$ are generated from a spray and have impact velocities varying between 60 and $80 \mathrm{~cm} / \mathrm{s}$. Impacts are observed from the side (using backlighting to enhance contrast) with a high-speed camera (Phantom V9) recording at 10000 frames per second. In all experiments, the origin of time $t$ is chosen when the distance between the bottom of the drop and the plate is minimal.

As seen in Fig. 1(b), a millimeter-sized oil drop impacting at $U=37 \mathrm{~cm} / \mathrm{s}$ a plate moving at $V=17 \mathrm{~m} / \mathrm{s}$ gets repelled, and the motion of the plate also induces an oblique rebound. Close to the disk, the liquid keeps a nonwetting shape at all times (also visible in the supplemental movie 1, see Ref. [22]), which indicates an absence of contact. Our aim is to describe the condition of repellency, together with the characteristics of the rebound-two properties that conspire to carry away the impacting liquid.

As shown by Lhuissier et al., a nonimpacting drop $(U \approx 0)$ can levitate if the plate velocity typically exceeds $1 \mathrm{~m} / \mathrm{s}$ [14]. However, impacts markedly modify the threshold of repellency: in Fig. 2(a) a millimeter-sized drop of silicone oil hitting at $U=0.3 \mathrm{~m} / \mathrm{s}$ a plate moving horizontally at $V=5 \mathrm{~m} / \mathrm{s}$ still contacts the plate, along which it gets entrained and stretched. In contrast, the same drop is repelled by a plate moving at $V=20 \mathrm{~m} / \mathrm{s} \mathrm{[Fig.} \mathrm{2(b)].} \mathrm{Oil} \mathrm{is} \mathrm{strongly} \mathrm{flattened} \mathrm{during} \mathrm{the}$ shock and then takes off obliquely after a time of about $3 \mathrm{~ms}$. As seen in Fig. 1(b), it bounces again when it reimpacts the substrate at a smaller velocity, and so on, until it gets swept by the moving plate, without leaving any visible trace.

The repellency threshold $V *$ is determined by decreasing the velocity $V$ until drops hitting the plate at a fixed speed $U$ get stuck. For $\eta>10 \mathrm{mPa}$ s, the transition is discontinuous: either the drop wets and elongates on the substrate $\left(V<V^{*}\right)$, as shown in movies 2 and 4 (Ref. [22]), or it fully bounces $\left(V>V^{*}\right)$, as illustrated in movie 3 (Ref. [22]). The abrupt character of the transition limits the error bars on the determination of $V^{*}$ to typically $\pm 1 \mathrm{~m} / \mathrm{s}$. After each experiment, the disk is cleaned with acetone to remove silicone oil (for $V<V^{*}$ ) or accidental contaminants (for $V>V^{*}$ ). Bouncing 

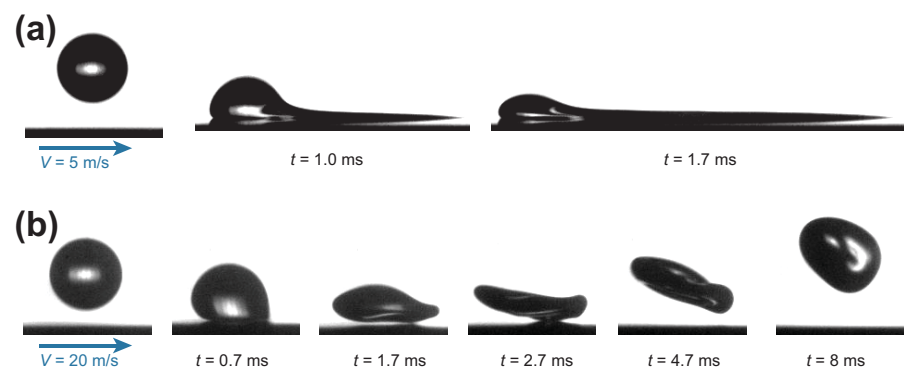

(c)

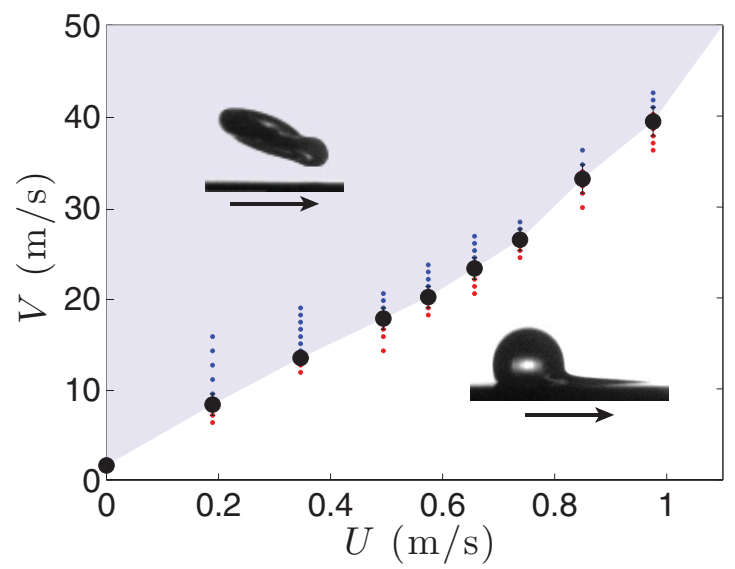

FIG. 2. Behavior of silicone oil $\left(R=0.9 \mathrm{~mm}, \eta=96 \mathrm{mPa} \mathrm{s}, \gamma=21 \mathrm{mN} / \mathrm{m}, \rho=960 \mathrm{~kg} / \mathrm{m}^{3}\right)$ impacting at a velocity $U$ a plate moving horizontally at a speed $V$. Origin of time $t$ is chosen when the oil-plate gap is minimum. (a) For $U=0.3 \mathrm{~m} / \mathrm{s}$ and $V=5 \mathrm{~m} / \mathrm{s}$, the drop contacts the solid and spreads. The corresponding movie is movie 2, see Ref. [22]. (b) For $U=0.3 \mathrm{~m} / \mathrm{s}$ and $V=20 \mathrm{~m} / \mathrm{s}$, the drop bounces and obliquely takes off. The corresponding movie is movie 3, see Ref. [22]. At such drop velocity $U$, bouncing occurs for $V>V^{*}=$ $11 \mathrm{~m} / \mathrm{s}$. (c) Phase diagram of repellency: red dots show when drops stick to the plate [as shown in Fig. 2(a)], while blue dots and the gray zone indicate that drops bounce [as shown in Fig. 2(b)]. Black circles mark the transition between contact and repellency. For each $U$, five to 10 series of experiments were performed to minimize the error bars.

and sticking behaviors are respectively reported in blue and red in Fig. 2(c), where the threshold $V^{*}$ is marked with black circles. As evidenced in Fig. 2(c), $V^{*}$ increases with $U$, which it strongly amplifies: $V^{*}$ is typically 30 to 40 times larger than $U$. For $U=1 \mathrm{~m} / \mathrm{s}$ for example, the plate must go faster than $40 \mathrm{~m} / \mathrm{s}$ to reflect the incoming oil. We now discuss the origin of this "amplification," and more generally what triggers repellency.

\section{THRESHOLD OF REPELLENCY}

We focus on the instants before impact. Figure 3(a) shows a drop of silicone oil $(R=0.91 \mathrm{~mm}$, $U=0.29 \mathrm{~m} / \mathrm{s})$ approaching $(t=-0.1 \mathrm{~ms})$ a plate moving at $V=38 \mathrm{~m} / \mathrm{s}$. The base of the drop is found to be asymmetrically flattened, in such a way that a wedge of air forms at its base, as also visible in movie 5 (see Ref. [22]). The same shape is found when using ethanol (movie 6, in Ref. [22]) or water (movie 7, in Ref. [22]) instead of silicone oil. We interpret this deformation as resulting from the strong dynamic pressure exerted on the oil by the air entrained by the moving plate. In Fig. 3(a) the viscous boundary layer of air has a thickness $\delta$ of $500 \mu \mathrm{m}$, a scale indicated by the dotted line and found to match the obliquely flattened part of the drop. The force generated by the impact of the boundary layer on this oblique interface is sketched in Fig. 3(a). Its vertical component 
(a)

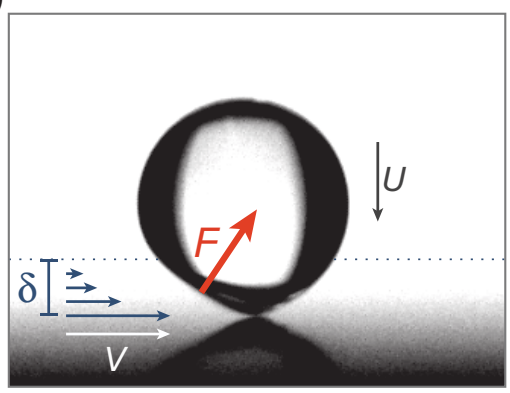

(b)

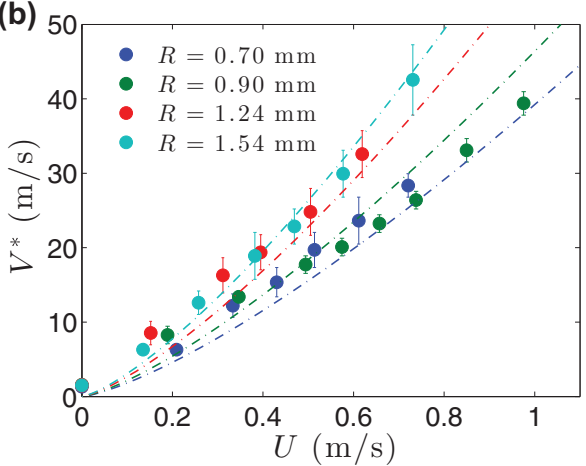

FIG. 3. (a) Drop of silicone oil $(R=0.91 \mathrm{~mm})$ at $t=-0.1 \mathrm{~ms}$ for $U=0.29 \mathrm{~m} / \mathrm{s}$ and $V=38 \mathrm{~m} / \mathrm{s}$. The drop is deformed by the lateral wind carried by the boundary layer whose thickness $\delta \approx 2.5\left(\eta_{\mathrm{a}} L / \rho_{\mathrm{a}} V\right)^{1 / 2}$ here is $500 \mu \mathrm{m}$ (dotted line). Air exerts a dynamic pressure, which reshapes the drop base. The induced force $F$ allows the drop to bounce. The corresponding movie is movie 5, see Ref. [22]. (b) Threshold velocity of repellency $V^{*}$ as a function of the impact velocity $U$, for oil drops with radius $R$ between 0.7 and $1.54 \mathrm{~mm}$. Dotted lines show Eq. (1), with numerical prefactor $\alpha=0.57$.

can lift up the liquid, allowing it to bounce, while the horizontal component participates to the drop entrainment along the plate.

The Reynolds number $\rho_{\mathrm{a}} V \delta / \eta_{\mathrm{a}}$ in air is typically between 100 and 1000 , so that the aerodynamic force $F$ exerted on the drop is inertial in nature. Contact with the solid can be prevented if the vertical component of the aerodynamic force overcomes the inertia of the impacting drop. On the one hand, and for $2 R>\delta$, the lifting force $F$ scales as the mean dynamic pressure of air within the boundary layer $\rho_{\mathrm{a}} V^{2}$ integrated over the surface area $\sim \delta R$ of the flattened part of the drop. This yields $F \sim \rho_{\mathrm{a}} V^{2} \delta R$-an expression different from that of Povarov who did not consider the formation of a wedge of air [1]. On the other hand, the liquid has to decelerate from $U$ to 0 in a time $R / U$, which implies an inertial force of order $\rho R^{3} U^{2} / R=\rho R^{2} U^{2}$ (a quantity that overcomes the drop weight). Rebounds happen if the latter force is smaller than $F$, that is, $V>\left(\rho R / \rho_{\mathrm{a}} \delta\right)^{1 / 2} U$. Both drop and boundary layer are typically millimeter-sized, which implies that the plate speed $V$ required for repellency must be at least $\left(\rho / \rho_{\mathrm{a}}\right)^{1 / 2} \approx 30$ times larger than the drop velocity $U$, as observed in Fig. 2(c). This "amplification" factor arises from the competition between two forms of inertia, that of the liquid and that of the air. As discussed earlier in the paper, $\delta$ itself is a function of $V$, from which we can deduce an explicit formula for the threshold velocity $V^{*}$ for drops larger than $\delta$ :

$$
V^{*} \sim\left(\frac{\rho^{2} R^{2}}{\rho_{a} \eta_{a} L}\right)^{1 / 3} U^{4 / 3} .
$$

The onset of repellency strongly depends on the impact speed $U$, as indeed observed in Fig. 2(c). Dependency is slightly stronger than linear, which can be tested by varying $U$ over a large range, as reported in Fig. 3(b) where dotted lines correspond to Eq. (1) with a unique fitting parameter $\alpha$ of 0.57 (of order unity). Lines are observed to fairly describe series of data with various radii $R$, and $V^{*}$ increases with the drop size in a way predicted by Eq. (1): large drops having more inertia than small ones need stronger airflows to get repelled. In addition, varying the drop viscosity $\eta$ by a factor 10 does not affect the scaling of $V^{*}$ with $U$ (in Ref. [22], Supplemental Fig. 1), even if it modestly impacts the prefactor $\alpha$ : decreasing $\eta$ by a factor 10 increases $\alpha$ by (only) 30\%: less viscous drops are slightly more challenging to repel. Strong shear flows within the boundary layer excite capillary waves at the drop surface (as seen in movie 6, Ref. [22]), which favors solid-liquid contact and thus slightly increases $V^{*}$. At large viscosity, these waves are expected to be damped, and we indeed observe in the supplementary Fig. 2 that $V^{*}$ becomes independent of $\eta$ for $\eta>300 \mathrm{mPas}$ (see Ref. [22]). 

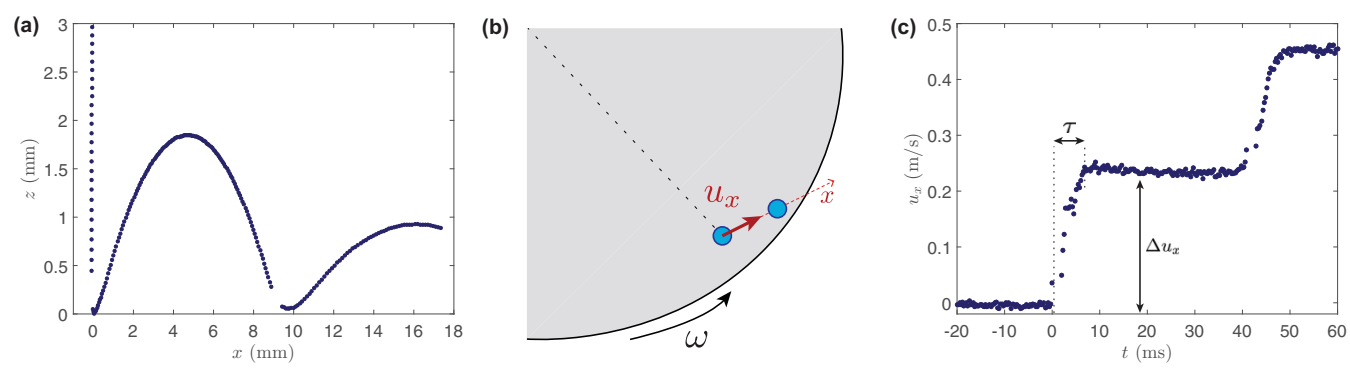

FIG. 4. (a) Side view of the center of mass of a $\operatorname{drop}(U=0.37 \mathrm{~m} / \mathrm{s}, R=0.75 \mathrm{~mm}, V=17 \mathrm{~m} / \mathrm{s})$ showing its first two rebounds. (b) Top view of the rotating disk: drops dispensed $1 \mathrm{~cm}$ from the edge of the disk move along an axis denoted as $x$ with a horizontal velocity $u_{x}$. (c) Velocity $u_{x}$ of oil as a function of time $t$. At each impact (with duration $\tau$ of about $6 \mathrm{~ms}$ ), the liquid is accelerated by an amount $\Delta u_{x} \sim 0.2 \mathrm{~m} / \mathrm{s}$.

Our scenario relies on the wedge deformation reported in Fig. 3(a) and supposed to be responsible for the lift-generating bouncing. The deformation of the drop requires conditions for both surface tension and viscosity. (1) The drop at the scale $\delta<R$ can be deformed only if the dynamical pressure of air overcomes surface tension effect. This can be written $\rho_{\mathrm{a}} V^{2} \delta R>\gamma \delta$, that is, $W e=\rho_{\mathrm{a}} V^{2} R / \gamma>1$, where the Weber number is unusually built with the air density. Focusing on the plate velocity, this criterion becomes $V>\left(\gamma / \rho_{\mathrm{a}} R\right)^{1 / 2}$. For our parameters, $V$ must be larger than typically $5 \mathrm{~m} / \mathrm{s}$, which it is in Fig. 3(a). According to this criterion, it is harder to repel water than oil or ethanol, as observed by Povarov et al. who reported a minimum velocity $V^{*}$ as high as $10 \mathrm{~m} / \mathrm{s}$ to repel water [1], of surface tension three times larger than that of oil. (2) Deformation must occur fast enough to avoid contact. The deformation time $\tau_{\mathrm{v}}$ is obtained by balancing the viscous force $\eta \delta R / \tau_{\mathrm{v}}$ with the aerodynamic force $\rho_{\mathrm{a}} V^{2} \delta R$. This yields $\tau_{\mathrm{v}} \sim \eta / \rho_{\mathrm{a}} V^{2}$, which we assume shorter than the crashing time $R / U$. For liquids of low viscosity, this condition is always satisfied. Rewritten as $\eta<\rho_{\mathrm{a}} V^{2} R / U$, it suggests that viscosity must be less than typically $1 \mathrm{~Pa}$ (quite a high value), as it is here. Repellency of more viscous oils such as shown in movie 8 (see Ref. [22]) will deserve a separate study.

By deforming the liquid and by generating a lift force, the boundary layer dramatically increases the natural "cushioning effect" of air. Millimeter-sized drops with velocities on the order of $1 \mathrm{~m} / \mathrm{s}$ are repelled, in stark contrast with immobile surfaces, either horizontal [23] or inclined [24], where drops can be reflected only if they exhibit normal velocities lower than typically $20 \mathrm{~cm} / \mathrm{s}$. Moving the surface changes the nature of the air cushion: while the force enabling rebounds on immobile plates has a viscous origin (it is generated by the $\mu \mathrm{m}$-thin layer of air flowing below the drop [23]), the repulsion becomes inertial for moving plates.

\section{DROP EVACUATION}

As for drops impacting tilted repellent plates [24,25], rebounds are not specular [Fig. 1(b)]. Drops after rebound take off obliquely due to the horizontal momentum gained at impact, before bouncing again when coming back to the plate. We extract from side views [in the $(x, z)$ plane] the drop trajectory. As shown in Fig. 4(a), $z(x)$ is observed to follow portions of parabola: successive impacts contribute to the evacuation of the liquid. As sketched in Fig. 4(b), we define $u_{x}=d x / d t$ as the horizontal drop velocity, in the plane of the disk. The evacuation speed $u_{x}(t)$ is found to increase at each impact by typically $\Delta u_{x} \approx 0.2 \mathrm{~m} / \mathrm{s}$, while it plateaus between two shocks, when the drop flies off the plate. The shocks (whose duration $\tau \approx 6 \mathrm{~ms}$ is indicated on the plot) are significantly shorter than the time spent out of the boundary layer, which gives to $u_{x}(t)$ a steplike shape.

The aerodynamic force sketched in Fig. 3(a) provides horizontal momentum at each impact, which entrains the liquid in the plate direction. For $\delta<2 R$, we can balance this force, $\rho_{\mathrm{a}} V^{2} \delta R$, with inertia, $\rho R^{3} \Delta u_{x} / \tau$. This yields a scaling law for the velocity increment, $\Delta u_{x} \sim \rho_{\mathrm{a}} V^{2} \delta \tau / \rho R^{2}$. Assuming $\tau \sim 2 R / U$, that is, the time necessary for the drop to stop, we get $\Delta u_{x} \sim \rho_{\mathrm{a}} V^{2} \delta / \rho R U$. For a plate 


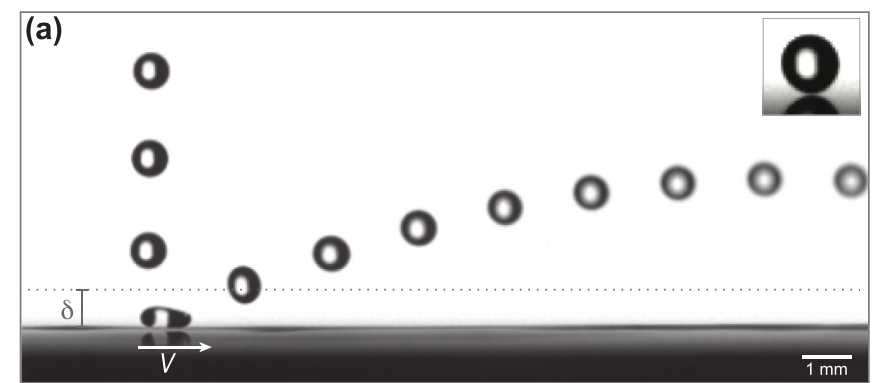

(b)

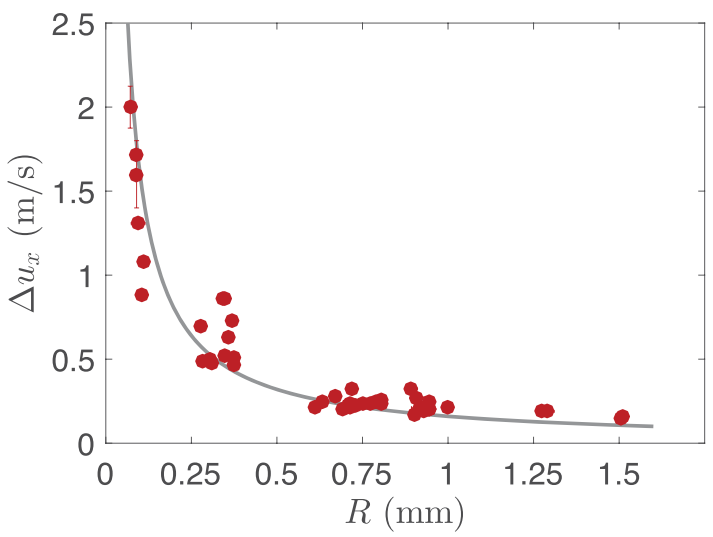

FIG. 5. (a) Images separated by $3.3 \mathrm{~ms}$ of a droplet of silicone oil $(R=0.34 \mathrm{~mm})$ hitting at $U=0.6 \mathrm{~m} / \mathrm{s}$ a plate moving at $V=17 \mathrm{~m} / \mathrm{s}$. In the limit $2 R<\delta=0.75 \mathrm{~mm}$, the velocity $\Delta u_{x}$ gained after the shock is much larger than previously, as seen from the increased distance between successive positions after take-off. $\Delta u_{x}$ here is $\sim 0.5 \mathrm{~m} / \mathrm{s}$, instead of $\sim 0.2 \mathrm{~m} / \mathrm{s}$ in Fig. 4 . The inset shows a zoomed-in picture of oil just before impact: even drops smaller than the boundary layer are asymmetrically deformed. The corresponding movie is movie 9 , see Ref. [22]. (b) Increment $\Delta u_{x}$ in horizontal velocity at impact as a function of $R$, for $U=0.44 \pm 0.16 \mathrm{~m} / \mathrm{s}$ and $V=17 \mathrm{~m} / \mathrm{s}$. $\Delta u_{x}$ can be fitted by a hyperbola (thin solid line) of equation $\Delta u_{x}=D / R$, with $D=1.6 \mathrm{~cm}^{2} / \mathrm{s}$, which we discuss in the text.

speed $V \approx 10 \mathrm{~m} / \mathrm{s}$, millimeter scales for boundary layers and drops and $\tau \approx 5 \mathrm{~ms}$, we get a jump in tangential velocity $\Delta u_{x} \approx 0.5 \mathrm{~m} / \mathrm{s}$, in agreement with observations. The interaction of the liquid with the air layer appears to be crucial not only for generating rebounds, but also for communicating horizontal momentum to impinging drops, which explains the nonspecular character of the rebounds.

\section{SPRAY REPELLENCY}

We can finally wonder whether droplets smaller than $\delta$ are similarly repelled or rather entrained by the plate, possibly adhering to it. The drop radius is decreased by using thinner needles, by spraying the oil (using a perfume sprayer) or by considering the satellite droplets produced by large drops detaching from the needle. The corresponding range of drop radii spans from $100 \mu \mathrm{m}$ to $1.5 \mathrm{~mm}$, which crosses the frontier $2 R=\delta$.

As seen in Fig. 5(a), small droplets can still bounce on moving plates: they again transiently form a wedge of air (inset of Fig. 5(a) and movie 9 in Ref. [22]), an effect we attribute to the ability of strong velocity gradients in the boundary layer to generate an asymmetry of the drop base. The rebound is more oblique than previously, owing to a larger speed increment $\Delta u_{x} . \Delta u_{x}$ quantifies the efficiency of evacuation, and it is found in Fig. 5(b) to increase dramatically as $R$ decreases. Tiny drops (with small inertia) are not only repelled, but also swept away by the plate. In the limit 
$2 R<\delta$, the dynamical quantities discussed earlier become explicit. On the one hand, the time $\tau$ during which momentum is gained by the impinging droplet is $2 \delta / U$ (the time spent in the boundary layer), which yields a momentum variation $\rho R^{3} \delta \Delta u_{x} / U$. On the other hand, the aerodynamic force on a drop fully immersed in the boundary layer scales as $\rho_{\mathrm{a}} V^{2} R^{2}$. The balance of these forces leads to $\Delta u_{x} \sim \rho_{\mathrm{a}} V^{2} \delta / \rho U R$, which, remarkably, is the same formula as for large drops. Indeed, a unique hyperbolic fit [full line in Fig. 5(b)] captures all the data, allowing us to understand the enhanced evacuation of small droplets $\left(\Delta u_{x} \sim D / R\right)$, contrasting with superhydrophobic repellency where they tend to stick [5]. The coefficient $D \sim \rho_{\mathrm{a}} V^{2} \delta / \rho U$ is expected to be $\sim 1 \mathrm{~cm}^{2} / \mathrm{s}$, comparable to $D=1.6 \pm 0.1 \mathrm{~cm}^{2} / \mathrm{s}$, the value providing the best fit of data in Fig. 5(b).

\section{CONCLUSION}

Dynamical repellency is unique because nonwetting then becomes independent of both solid and liquid: even wetting and viscous liquids can be repelled that way. Hence controlling the airflow around a moving solid (or vehicle) might in principle keep it dry under impacting rain or projections, provided we satisfy the bouncing conditions. Repellency is observed when the plate velocity is larger than a critical value $V^{*}$ increasing with the impact speed $U$ [Eq. (1)]. At high $U, V^{*}$ can be so large that the boundary layer becomes turbulent, which induces a jump in $V^{*}[1]$ that remains to be understood. We assume that rebound arises from the presence of an asymmetric wedge of air sculpted by the dynamic pressure of the air carried by the plate. Being perpendicular to the wedge, the pressure force has a vertical component that can lift off the liquid and induce rebounds. (As a consequence, solid particles cannot be repelled that way, which we checked using millimeter-sized polystyrene marbles.) More generally, dynamical repellency might be seen as a kind of Leidenfrost effect, where the control parameter is velocity instead of temperature; in both cases, the sensitivity towards the speed of impacting liquids is expressed by a shift of this control parameter [11]. However, unlike in the Leidenfrost effect, the solid motion induces oblique rebounds. This enhances the evacuation of liquid, found to be not only reflected but also pushed horizontally away from the solid, and even more efficiently when drops are small.

\section{ACKNOWLEDGMENTS}

We thank Direction Générale de l'Armement (DGA) for financial support. We also thank Mathieu Bancelin, Jacy Bird, Samuel Cristiani, and Mayuko Murano for preliminary experiments on this subject.

[1] O. A. Povarov, O. I. Nazarov, L. A. Ignat'evskaya, and A. I. Nikol'skii, Interaction of drops with boundary layer on rotating surface, J. Eng. Phys. Thermophys. 31, 1453 (1976).

[2] G. S. Hartley and R. T. Brunskill, Reflection of water drops from surfaces, in Surface Phenomena in Chemistry and Biology, edited by J. F. Danielli (Pergamon Press, Oxford, 1958), p. 214.

[3] K. M. Wisdom, J. A. Watson, X. Qu, F. Liu, G. S. Watson, and C. H. Chen, Self-cleaning of superhydrophobic surfaces by self-propelled jumping condensate, Proc. Natl. Acad. Sci. USA, 110, 7992 (2013).

[4] R. Blossey, Self-cleaning surfaces-Virtual realities, Nat. Mater. 2, 301 (2003).

[5] D. Richard and D. Quéré, Bouncing water drops, Europhys. Lett. 50, 769 (2000).

[6] J. C. Bird, R. Dhiman, H. M. Kwon, and K. K. Varanasi, Reducing the contact time of a bouncing drop, Nature (London) 503, 385 (2013).

[7] A. Tuteja, W. Choi, J. M. Mabry, G. H. McKinley, and R. E. Cohen, Robust omniphobic surfaces, Proc. Natl. Acad. Sci. USA 105, 18200 (2008).

[8] T. Vasileioua, J. Gerbera, J. Prautzscha, T. M. Schutziusa, and D. Poulikakos, Superhydrophobicity enhancement through substrate flexibility, Proc. Natl. Acad. Sci. USA 113, 13307 (2016). 
[9] X. Deng, L. Mammen, H. J. Butt, and D. Vollmer, Candle soot as a template for a transparent robust superamphiphobic coating, Science 335, 67 (2012).

[10] J. D. Bernardin, C. J. Stebbins, and I. Mudawar, Mapping of impact and heat transfer regimes of water drops impinging on a polished surface, Int. J. Heat Mass Transfer 40, 247 (1997).

[11] T. Tran, H. J. J. Staat, A. Prosperetti, C. Sun, and D. Lohse, Drop Impact on Superheated Surfaces, Phys. Rev. Lett. 108, 036101 (2012).

[12] Y. Couder, E. Fort, C. H. Gautier, and A. Boudaoud, From Bouncing to Floating: Noncoalescence of Drops on a Fluid Bath, Phys. Rev. Lett. 94, 177801 (2005).

[13] T. Gilet and J. W. M. Bush, The fluid trampoline: Droplets bouncing on a soap film, J. Fluid Mech. 625, 167 (2009).

[14] H. Lhuissier, Y. Tagawa, Y. Tran, and C. Sun, Levitation of a drop over a moving surface, J. Fluid Mech. 733, R4 (2013).

[15] A. Gauthier, J. C. Bird, C. Clanet, and D. Quéré, Aerodynamic Leidenfrost effect, Phys. Rev. Fluids 1, 084002 (2016).

[16] K. R. Sreenivas, P. K. De, and J. H. Arakeri, Levitation of a drop over a film flow, J. Fluid Mech. 380, 297 (1999).

[17] A. Duchesne, C. Savaro, L. Lebon, C. Pirat, and L. Limat, Multiple rotations of a drop rolling inside a horizontal circular hydraulic jump, Europhys. Lett. 102, 64001 (2013).

[18] T. v. Kármán, Über laminare und turbulente Reibung, Z. Angew. Math. Mech. 1, 233 (1921).

[19] N. Gregory, J. T. Stuart, and W. S. Walker, On the stability of three-dimensional boundary layers with application to the flow due to a rotating disk, Philos. Trans. R. Soc. A 248, 155 (1955).

[20] R. J. Lingwood, An experimental study of absolute instability of the rotating-disk boundary-layer flow, J. Fluid Mech. 314, 373 (1996).

[21] M. E. Siddiqui, V. Mukund, J. Scott, and B. Pier, Experimental characterization of transition region in rotating-disk boundary layer, Phys. Fluids. 25, 034102 (2013).

[22] See Supplemental Material at http://link.aps.org/supplemental/10.1103/PhysRevFluids.3.054002 for supplemental Figs. 1 and 2 - on the influence of oil viscosity on the threshold of repellency, and to access movies 1 to 9 as well as the corresponding legends.

[23] Š. Šikalo, C. Tropea, and E. N. Ganić, Impact of droplets onto inclined walls, J. Colloid Interface Sci. 286, 661 (2005).

[24] J. de Ruiter, R. Lagraauw, D. van den Ende, and F. Mugele, Wettability-independent bouncing on flat surfaces mediated by thin air film, Nat. Phys. 11, 48 (2015).

[25] Y. H. Yeong, J. Burton, E. Loth, and I. S. Bayer, Drop impact and rebound dynamics on an inclined superhydrophobic surface, Langmuir 30, 12027 (2014). 